This is the author's final, peer-reviewed manuscript as accepted for publication. The publisher-formatted version may be available through the publisher's web site or your institution's library.

\title{
Stem rust resistance in Aegilops tauschii germplasm
}

Matthew N. Rouse, Eric L. Olson, Bikram S. Gill, Michael O. Pumphrey, Yue Jin

\section{How to cite this manuscript}

If you make reference to this version of the manuscript, use the following information:

Rouse, M.N., Olson, E.L., Gill, B.S., Pumphrey, M.O., \& Jin, Y. (2011). Stem rust resistance in Aegilops tauschii germplasm. Retrieved from http://krex.ksu.edu

\section{Published Version Information}

Citation: Rouse, M.N., Olson, E.L., Gill, B.S., Pumphrey, M.O., \& Jin, Y. (2011). Stem rust resistance in Aegilops tauschii germplasm. Crop Science, 51(5), 2074-2078.

Copyright: Copyright @ 2011. Copyright @ by the Crop Science Society of America, Inc

Digital Object Identifier (DOI): doi: 10.2135/cropsci2010.12.0719

Publisher's Link: https://www.crops.org/publications/cs/articles/51/5/2074

This item was retrieved from the K-State Research Exchange (K-REx), the institutional repository of Kansas State University. K-REx is available at http://krex.ksu.edu 


\section{Stem rust resistance in Aegilops tauschii germplasm}

Matthew N. Rouse, Eric L. Olson, Bikram S. Gill, Michael O. Pumphrey, and Yue Jin*

Mathew N. Rouse and Yue Jin, USDA-ARS Cereal Disease Laboratory, Department of Plant Pathology, University of Minnesota, St. Paul, MN 55108. Eric L. Olson and Bikram Gill, Department of Plant Pathology, Kansas State University, Manhattan, KS 66506. Michael O. Pumphrey, Department of Crop and Soil Sciences, Washington State University, Pullman, WA 99164.

*Corresponding author Email: Yue.Jin@ ars.usda.gov

Abbreviations: CSIRO, Commonwealth Scientific and Industrial Research Organization; ID, Idaho; IT, Infection type; KS, Kansas; MN, Minnesota; Pgt, Puccinia graminis f. sp. tritici; US, United States; USDA, United States Department of Agriculture; ARS, Agricultural Research Service; TRTTF, TTKSK, TTTTF, QTHJC, RKQQC, TPMKC, QFCSC, and MCCFC, Pgt races

Mention of trade names or commercial products in this article is solely for the purpose of providing specific information and does not imply recommendation or endorsement by the U.S. Department of Agriculture. 


\begin{abstract}
Aegilops tauschii Cosson, the D genome donor of hexaploid wheat, Triticum aestivum L., has been used extensively for the transfer of agronomically important traits to wheat, including stem rust resistance genes $\mathrm{Sr} 33, \mathrm{Sr} 45$, and $\mathrm{Sr} 46$. In order to identify potentially new stem rust resistance genes in A. tauschii germplasm, we evaluated 456 nonduplicated accessions deposited in the USDA National Small Grains Collection (Aberdeen, ID) and the Wheat Genetic and Genomic Resources Center collection (Kansas State University, Manhattan, KS), with races TTKSK (Ug99), TRTTF, TTTTF, TPMKC, RKQQC, and QTHJC of Puccinia graminis Pers.:Pers. f. sp. tritici Eriks. \& E. Henn. Ninety-eight accessions (22\%) were identified as resistant to race TTKSK. A broad range of resistant infection types (; to $2+)$ were found in reaction to race TTKSK. Resistance was significantly associated among most of the races in pair-wise comparisons. However, resistance was largely race-specific. Only 12 of the accessions resistant to race TTKSK were also resistant to the other five races. Results from this germplasm screening will facilitate further studies on the genetic characterization of accessions with potentially novel sources of resistance to race TTKSK.
\end{abstract}




\section{Introduction}

Aegilops tauschii Cosson, is the donor of the D genome of hexaploid wheat (Triticum aestivum L.) (Kihara, 1944; McFadden and Sears, 1946). Wheat stem rust, caused by Puccinia graminis Pers.:Pers f. sp. tritici Eriks. \& E. Henn. (Pgt), has historically been a major wheat yield-limiting factor. The close relationship between $A e$. tauschii and T. aestivum has facilitated the rapid introgression of several agronomically important disease traits from Ae. tauschii to T. aestivum including resistance to wheat stem rust (Kerber and Dyck, 1979; Gill and Raupp, 1987; Marais et al., 1998).

Recent epidemics of stem rust in Eastern Africa have raised concern about the resistance of currently grown wheat cultivars to new stem rust races from Africa. In 1999, $P g t$ was reported from Uganda that possessed virulence to the majority of stem rust resistance genes used in agriculture (Pretorius et al., 2000; Jin et al., 2007). Isolates were designated as race TTKSK (or Ug99) based upon the North American stem rust nomenclature (Jin et al., 2008). Race TTKSK and/or variants have spread throughout eastern and southern Africa, Yemen, and Iran (Nazari et al., 2009; Pretorius et al., 2010; Singh et al., 2006). Variants of race TTKSK have been identified with additional virulence to resistance genes $\operatorname{Sr} 24$ and $\operatorname{Sr} 36$ (Jin et al., 2008; Jin et al., 2009). These variants (races TTKST and TTTSK, respectively) pose an even greater threat to worldwide wheat production. Screening of currently grown cultivars and breeding germplasm indicated that the majority of the germplasm from Asia, the United States, and Canada are susceptible to TTKSK (Fetch 2007; Jin and Singh, 2006; Singh et al., 2008). Unfortunately, much of the resistance to race TTKSK available in the United States is conferred by $\mathrm{Sr} 24$ and $\mathrm{Sr} 36$ (this resistance is not effective to races TTKST and 
TTTSK). Of the available sources of resistance to race TTKSK and variants described todate, most of the resistance genes have been introgressed from wild relatives of wheat (Jin et al., 2007) and have not been utilized extensively in agriculture because of linkage between these genes and deleterious factors (Singh et al., 2008).

Three stem rust resistance genes have been transferred from Ae. tauschii to wheat previously: Sr33, Sr45, and Sr46 (Kerber and Dyck, 1979; Marais et al., 1998; Lagudah, unpublished). These genes provide resistance to race TTKSK (Jin et al., 2007; Rouse and Jin unpublished). Additional resistance genes may be present in Ae. tauschii germplasm. The identification of new genes will provide breeders with diverse genes for pyramiding in order to increase the durability of resistance. Our objective was to screen the available accessions of Ae. tauschii for resistance to race TTKSK in order to facilitate the characterization and introgression of novel resistance. 


\section{Materials and Methods}

Aegilops tauschii accessions were obtained from the USDA-ARS National Small Grains Collection (Aberdeen, ID, 118 accessions) and from the Wheat Genetic and Genomic Resources Center (Manhattan, KS, 412 accessions). The accession names and sources were cross-checked in order to eliminate accessions that were redundant among or within the two collections. We identified 456 non-redundant accessions. The geographic origins of these accessions are displayed in Table 1.

Six to ten seedlings of the 456 accessions were inoculated with six races of Pgt: TRTTF, TTKSK, TTTTF, QTHJC, RKQQC, and TPMKC (Table 2). Screening with TRTTF, TTKSK, and TTTTF was conducted at the USDA-ARS Cereal Disease Laboratory (Saint Paul, MN). Screening with QTHJC, RKQQC, and TPMKC was conducted at Kansas State University (Manhattan, KS). Accessions that have been used previously to introgress stem rust resistance genes Sr33 and Sr45, RL5288 and RL5289, respectively, were obtained from Colin Heibert (Agriculture and Agri-Food Canada, Winnipeg, Manitoba). The diploid Ae. tauschii source of Sr46 (AUS 18913) and genetic stocks of Sr33 and Sr45 in hexaploid backgrounds were obtained from CSIRO (CSID 5404 and CSID 5406, respectively). These stocks and susceptible Chinese Spring (CI 14108) were inoculated with the six $P g t$ races. Urediniospores of stem rust isolates in gelatin capsules stored at $-80^{\circ} \mathrm{C}$ were heat-shocked at $45^{\circ} \mathrm{C}$ for $15 \mathrm{~min}$, and placed in a rehydration chamber for 2 to $4 \mathrm{~h}$ maintained at $80 \%$ relative humidity by a $\mathrm{KOH}$ solution (Rowell, 1984). Procedures in inoculation, incubation and disease assessment were as described previously (Jin et al., 2007). Infection types (ITs) were classified as in Stakman 
et al. (1962). Infection types with substantial necrosis or chlorosis were designated as "N" or "C", respectively. Low infection frequency (LIF) was used to indicate notably low density of uredinia for a given leaf area. ITs 0 to $2++$ were considered low ITs indicating host resistance whereas ITs $3=$ to 4 were considered high ITs indicating host susceptibility. When low and high ITs were present on the same leaf, the plant was considered resistant. Accessions were classified as heterogeneous when both resistant and susceptible plants were present.

Frequencies of resistant, susceptible, and heterogeneous accessions were calculated for each of the six races. For each accession, the reaction to the combined races was determined as susceptible if the IT to any of the six races was high, resistant if the ITs to all six races was low, and heterogeneous if the reaction to one of the races was heterogeneous and the reaction to the five other races was low or heterogeneous. In order to test for associations of resistance, we calculated $\chi^{2}$ values based on the assumption of independence of resistance to each race for every pair-wise comparison of races. Percent of accessions resistant or heterogeneous for each country of origin were calculated.

To measure the repeatability of visually scoring infection types, a total of 37 randomly selected accessions were planted a second time for screening with Pgt races TTTTF, TTKSK, and TRTTF for a second biological replication. This resulted in data available for 100 infection types pairs (poor germination limited the number of infection type pairs). Out of these 100 pairs, 7 were inconsistently recorded as resistant $(0, ;, 1$, or 2 ) in one replication and susceptible ( 3 or 4 ) in the other. The reason for inconsistencies was likely due to heterogeneity in accessions or error in visually scoring infection types. A total of 93 of the infection type pairs were consistent representing a repeatability of 
classifying resistance/susceptibility of $93 \%$. Similarly, we previously found the repeatability of classifying resistance/susceptibility for the diploid wheat relative Triticum monococcum (L.) to be greater than 95\% (Rouse and Jin, 2011). 


\section{Results}

The seedling infection types of the Ae. tauschii accessions are available as supplementary table S1. The frequencies of accessions resistant, susceptible, and heterogeneous to the six races are displayed in Table 3. Ninety-eight accessions (22.2\%) were resistant to race TTKSK, but only 12 of these accessions (2.7\%) were resistant to the five other races as well. Race TRTTF was the most virulent race within this germplasm $(88.2 \%$ of the accessions were susceptible) whereas QTHJC was the most avirulent (68.0\% of the accessions were susceptible). Relatively few of the accessions were heterogeneous to any of the races $(0.5 \%$ to $2.1 \%)$.

The diploid genetic stocks of Sr33, Sr45, and Sr46 (RL5288, RL5289, and AUS 18913, respectively) displayed unique infection type patterns to the races used (Table 4). The diploid source of $S r 46$ was also included in the germplasm screening (TA 1703, synonymous with AUS 18913). Variation in the Sr46 lines in reaction to races QTHJC and RKQQC may be attributed to (1) an unstable intermediate reaction to these races and/or (2) the screening of TA 1703 and AUS 18913 by different individuals in different locations (E. L. Olson, Manhattan, KS; M. N. Rouse, St. Paul, MN).

Significant positive associations were found for all race pair-wise comparisons except for races TTTTF and RKQQC (Table 5). However, diverse IT patterns were observed indicating the presence of multiple race-specific resistance genes.

Resistance was not equally distributed among countries of origin (Table 1). The 12 accessions resistant to the six Pgt races were distributed only among Azerbaijan, Iran, Turkmenistan, Uzbekistan, and of unknown origin. These countries of origin also had 
higher percentages of accessions resistant to race TTKSK compared to the other countries, with the exception of Kazakhstan where five of eight accessions were resistant to race TTKSK. 


\section{Discussion}

Previous studies have examined collections of Ae. tauschii or synthetic hexaploid wheats created with Ae. tauschii accessions for resistance to stem rust (Cox et al., 1992; Assefa and Fehrmann, 2004; Friesen et al., 2008; Ogbonnaya et al., 2008). We evaluated a relatively large collection of Ae. tauschii accessions in an attempt to identify resistance to race TTKSK and other races with broad virulence.

Accessions resistant to the six Pgt races were confined to five geographic origin classifications. Cox et al. (1992) found that resistance in a subset of these accessions to Pgt race TNM was confined to Caspian Iran or Azerbaijan. We confirmed that these countries possess a high frequency of resistance to multiple races of Pgt. The higher number of accessions and wider geographic range of germplasm tested in our study identified Turkmenistan as an additional hotspot for Pgt resistance.

In a survey for worldwide virulence in Pgt (Huerta-Espino, 1992), isolates with virulence to Sr33 have not been found. Seedling and adult plant resistance of hexaploid monogenic lines carrying Sr33 to race TTKSK is only moderate (Jin et al., 2007). The dilution of Ae. tauschii resistance when expressed in hexaploids (Kerber and Dyck, 1979) may be one reason why $S r 33$ resistance to race TTKSK is moderate. The adult plant resistance to race TTKSK conferred by $\mathrm{Sr} 45$ and $\mathrm{Sr} 46$ is unknown. $\mathrm{Sr} 45$ is ineffective to the predominant races in North America (Table 4; Kerber and Dyck, 1979; Marais et al., 1998). Our data indicate that $S r 46$ resistance is race-specific (Table 4). If these genes are used to provide resistance to race TTKSK, additional resistance genes will be necessary to provide resistance to other Pgt races. 
We interpret the significant positive associations of resistance to various races observed as evidence for genes conferring resistance to multiple races. However, only 12 accessions were resistant to the six races. Diverse IT patterns were observed among the Ae. tauschii accessions. The diversity of IT patterns prevented us from being able to confidently postulate the presence of known genes in the germplasm screened and likely indicate diversity for resistance genes, modifier genes, or genetic background effects. The IT patterns of a subset of accessions with unknown genes are displayed in Table 4. Many IT patterns could not be accounted for by the previously described genes alone or in combination. This suggests that additional stem rust resistance genes are likely present in Ae. tauschii germplasm. As Sr33 provides resistance to all six races, further studies are needed to test the allelic relationship between resistance in the 12 combined resistant accessions and Sr33. Preliminary evidence based on ITs suggest that accessions with resistance to all races screened possess a gene (or genes) independent of $\mathrm{Sr} 33$. For example, accession TA 10171 exhibited resistance to the six races with ITs much lower than observed on RL5288 (Table 4). Further studies testing the allelic relationship between previously described genes and potentially novel resistance are needed to confirm the presence of novel resistance to race TTKSK specifically. We have initiated crosses to confirm the presence of novel resistance to race TTKSK, to map potentially novel sources of resistance, and to introgress such resistance into adapted wheat backgrounds. 


\section{References}

Assefa, S., and H. Fehrmann. 2004. Evaluation of Aegilops tauschii Coss. for resistance to wheat stem rust and inheritance of resistance genes in wheat. Genetic Resources and Crop Evolution 51:663-669.

Cox, T. S., W. J. Raupp, D. L. Wilson, B. S. Gill, S. Leath, W. W. Bockus, and L. E. Browder. 1992. Resistance to foliar diseases in a collection of Triticum tauschii germ plasm. Plant Dis. 76:1061-1064.

Fetch, T. Jr. 2007. Virulence of stem rust race TTKS on Canadian wheat cultivars. Can. J. Plant Pathol. 29:441.

Friesen, T. L., S. S. Xu, and M. O. Harris. 2008. Stem rust, tan spot, Stagonospora nodorum blotch, and hessian fly resistance in Langdon durum-Aegilops tauschii synthetic hexaploid wheat lines. Crop Sci. 48:1062-1070.

Gill, B. S., and W. J. Raupp. (1987). Direct genetic transfers from Aegilops squarrosa L. to hexaploid wheat. Crop Sci. 27:445-450.

Huerta-Espino, J. 1992. Analysis of wheat leaf and stem rust virulence on a worldwide basis. PhD Thesis, University of Minnesota, USA.

Jin., Y., and R. P. Singh. 2006. Resistance in U.S. wheat to recent Eastern African isolates of Puccinia graminis f. sp. tritici with virulence to resistance gene $\operatorname{Sr} 31$. Plant Dis. 90:476-480.

Jin, Y., R. P. Singh, R. W. Ward, R. Wanyera, M. Kinyua, P. Njau, T. Fetch, Z. A. Pretorius, and A. Yahyaoui. 2007. Characterization of seedling infection types 
and adult plant infection responses of monogenic $S r$ gene lines to race TTKS of Puccinia graminis f. sp. tritici. Plant Dis. 91:1096-1099.

Jin, Y., L. J. Szabo, Z. A. Pretorius, R. P. Singh, R. Ward, and T. Fetch Jr. 2008. Detection of virulence to resistance gene $\mathrm{Sr} 24$ within race TTKS of Puccinia graminis f. sp. tritici. Plant Dis. 92:923-926.

Jin, Y., L. J. Szabo, M. N. Rouse, T. Fetch Jr., Z. A. Pretorus, R. Wanyera, and P. Njau. 2009. Detection of virulence to resistance gene $\operatorname{Sr} 36$ within the TTKS race lineage of Puccinia graminis f. sp. tritici. Plant Dis. 93:367-370.

Kihara, H. 1944. Discovery of the DD analyzer, one of the ancestors of Triticum vulgare. Agriculture and Horticulture 19:889-890.

Kerber, E. R., and P. L. Dyck. 1979. Resistance to stem and leaf rust of wheat in Aegilops squarrosa and transfer of a gene for stem rust resistance to hexaploid wheat. P. 358-364. In: S. Ramanujam (ed.) Proc. Int. Wheat Genet. Symp., $5^{\text {th }}$, New Delhi, India. 23-28 Feb. 1978. Indian Society of Genetics and Plant Breeding, New Delhi, India.

Marais, G. F., W. G. Wessels, M. Horn, and F. du Toit. 1998. Association of a stem rust resistance gene (Sr45) and two Russian wheat aphid resistance genes ( $D n 5$ and Dn7) with mapped structural loci in common wheat. S. Afr. J. Plant Soil 15:6771.

McFadden, E. S., and E. R. Sears. 1946. The origin of Triticum spelta and itsfree- threshing hexaploid relatives. J. Hered. 37:107-116. 
Nazari, K., M. Mafi, A. Yahyaoui, R. P. Singh, and R. F. Park. 2009. Detection of wheat stem rust (Puccinia graminis f. sp. tritici) race TTKSK (Ug99) in Iran. Plant Dis. 93:317.

Pretorius, Z. A., C. M. Bender, B. Visser, and T. Terefe. 2010. First report of a Puccinia graminis f. sp. tritici race virulent to the $\mathrm{Sr} 24$ and $\mathrm{Sr} 31$ wheat stem rust resistance genes in South Africa. Plant Dis. 94:784.

Pretorius, Z. A., R. P. Singh, W. W. Wagoire, and T. S. Payne. 2000. Detection of virulence to wheat stem rust resistance gene Sr31 in Puccinia graminis f. sp. tritici in Uganda. Plant Dis. 84:203.

Ogbonnaya, F. C., M. Imtiaz, H. S. Bariana, M. McLean, M. M. Shankar, G. J. Hollaway, R. M. Trethowan, E. S. Lagudah, and M. van Ginkel. 2008. Mining synthetic hexaploids for multiple disease resistance to improve bread wheat. Austr. J.Agric. Res. 59:421-431.

Rouse, M. N., and Y. Jin. 2011. Stem rust resistance in A-genome diploid relatives of wheat. Plant Dis. DOI: 10.1094/PDIS-04-10-0260.

Rowell, J. B. 1984. Controlled infection by Puccinia graminis f. sp. tritici under artificial conditions. Pages 292-332 in: W. R. Bushnell and A. P. Roelfs (eds.) The Cereal Rusts, Vol. 1. Origins, Specificity, Structure, and Physiology. Academic Press, Orlando, FL.

Singh, R. P., D. P. Hodson, J. Huerta-Espino, Y. Jin, P. Njau, R. Wanyera, S. A. HerreraFoessel, and R. Ward. 2008. Will stem rust destroy the world's wheat crop? Adv. Agron. 98:271-309. 
Singh, R. P., D. P. Hodson, Y. Jin, J. Huerta-Espino, M. G. Kinyua, R. Wanyera, P. Njau, and R. Ward. 2006. Current status, likely migration and strategies to mitigate the threat to wheat production from race Ug99 (TTKS) of stem rust pathogen. CAB Reviews: Perspectives in Agriculture, Veterinary Science, Nutrition and Natural Resources. 1 (No. 054).

Stakman, E. C., D. M. Steward, and W. Q. Loegering. 1962. Identification of physiologic races of Puccinia graminis var. tritici. United States Department of Agriculture Agricultural Research Service E-617. 


\section{Acknowledgements}

We would like to acknowledge Harold Bockelman, USDA-ARS, National Small Grains

Collection, Aberdeen, Idaho, for distributing Aegilops tauschii seed; Colin Heibert, Agriculture and Agri-Food, Canada for providing RL5288 and RL5289; Robert Bowden, USDA-ARS, Manhattan, Kansas for maintaining Pgt cultures; Jon Raupp, Lucy Wanschura and Sam Gale for technical assistance. 
Table 1. Geographic origin of Aegilops tauschii accessions and percentage of accessions resistant or heterogeneous to six Puccinia graminis f. sp. tritici races.

\section{Percent Resistant or Heterogeneous}

\begin{tabular}{lrrrrrrr} 
Country & Accessions & TRTTF & \multicolumn{1}{l}{ TTKSK } & TTTTF & QTHJC & RKQQC & TPMK \\
\hline Afghanistan & 83 & 1 & 6 & 0 & 4 & 17 \\
Armenia & 21 & 5 & 24 & 24 & 40 & 30 \\
Azerbaijan & 42 & 33 & 48 & 57 & 78 & 41 \\
China & 18 & 0 & 0 & 0 & 6 & 18 \\
Georgia & 14 & 0 & 14 & 7 & 43 & 29 \\
Iran & 64 & 35 & 43 & 20 & 52 & 39 \\
Kazakhstan & 8 & 0 & 63 & 38 & 100 & 100 \\
Kyrgyzstan & 4 & 0 & 25 & 0 & 0 & 75 \\
Pakistan & 10 & 0 & 22 & 0 & 0 & 10 \\
Syria & 5 & 0 & 20 & 0 & 20 & 20 \\
Tajikistan & 41 & 0 & 12 & 3 & 15 & 13 \\
Turkey & 30 & 4 & 14 & 4 & 8 & 4 \\
Turkmenistan & 45 & 16 & 18 & 22 & 31 & 48 \\
Unknown & 36 & 17 & 34 & 22 & 48 & 23 \\
Uzbekistan & 30 & 3 & 27 & 17 & 34 & 37 \\
\hline & & & & & &
\end{tabular}


Table 2. Races of Puccinia graminis f. sp. tritici used to screen Aegilops tauschii germplasm

\begin{tabular}{lllll}
\hline Isolate & Race & Origin & Avirulence & Virulence \\
\hline 04KEN156/04 & TTKSK $\dagger$ & Kenya & $2436 \mathrm{Tmp}$ & $567 b 8 a 9 a 9 b 9 d 9 e 9 g 101117$ \\
06YEM34-1 & TRTTF & Yemen & $8 a 2431$ & $567 b 9 a 9 b 9 d 9 e 9 g 10111721$ \\
01MN84A-1-2 & TTTTF & US & 2431 & $567 b 8 a 9 a 9 b 9 d 9 e 9 g 101117$ \\
75ND717C & QTHJC & US & $7 b 9 a 9 e 2430313638 \mathrm{Tmp}$ & $568 a 9 b 9 d 9 g 10111721 \mathrm{McN}$ \\
99KS76A-1 & RKQQC & US & $9 e 10111724303138 \mathrm{Tmp}$ & $567 b 8 a 9 a 9 b 9 d 9 g 2136 \mathrm{McN}$ \\
74MN1409 & TPMKC & US & $69 a 9 b 24303138$ & $57 b 8 a 9 d 9 e 9 g 1011172136 \mathrm{Tn}$ \\
\hline
\end{tabular}


Table 3. Number (and frequency) of Aegilops tauschii accessions resistant, susceptible, and heterogeneous to six races of Puccinia graminis f. sp. tritici and the combined reaction to all races

\begin{tabular}{lrrrrrrr}
\hline Race & \multicolumn{1}{c}{ Total } & \multicolumn{2}{c}{ Resistant (\%) } & \multicolumn{3}{c}{ Susceptible (\%) } & Heterogeneous (\%) \\
\hline TRTTF & 439 & 48 & $(10.9 \%)$ & 387 & $(88.2 \%)$ & 4 & $(0.91 \%)$ \\
TTKSK & 442 & 98 & $(22.2 \%)$ & 338 & $(76.5 \%)$ & 6 & $(1.36 \%)$ \\
TTTTF & 436 & 61 & $(14.0 \%)$ & 366 & $(83.9 \%)$ & 9 & $(2.06 \%)$ \\
QTHJC & 413 & 130 & $(31.5 \%)$ & 281 & $(68.0 \%)$ & 2 & $(0.48 \%)$ \\
RKQQC & 422 & 121 & $(28.7 \%)$ & 299 & $(70.9 \%)$ & 2 & $(0.47 \%)$ \\
TPMKC & 423 & 95 & $(22.5 \%)$ & 326 & $(77.1 \%)$ & 2 & $(0.47 \%)$ \\
All Races & 448 & 12 & $(2.68 \%)$ & 432 & $(96.4 \%)$ & 4 & $(0.89 \%)$ \\
\hline
\end{tabular}


Table 4. Infection types of Aegilops tauschii accessions with known stem rust resistance genes and selected lines with unknown genes

\begin{tabular}{|c|c|c|c|c|c|c|}
\hline Accession & Background & Gene & TRTTF & TTKSK & TTTTF & QTHJC \\
\hline $\begin{array}{l}\text { Chinese } \\
\text { Spring }\end{array}$ & T. aestivum & - & $4 \uparrow$ & 4 & 4 & 3 \\
\hline RL5288 & Ae. tauschii & Sr33 & - & - & $2 / 2,2+$ & 2 \\
\hline CSID 5405 & T. aestivum & Sr33 & - & $2++$ & - & $2,2-$ \\
\hline RL5289 & Ae. tauschii & Sr45 & - & - & 4 & 4 \\
\hline CSID 5406 & T. aestivum & Sr45 & 4 & 0 ; & 4 & 3 \\
\hline TA 1703 & Ae. tauschii & Sr46 & 2 & ;,2- & 4 & $2-$ \\
\hline AUS 18913 & Ae. tauschii & Sr46 & 2 & ;1,2- & $3+$ & 3 \\
\hline TA 10087 & Ae. tauschii & - & 1 & ;2- & $;, 1 \mathrm{C}$ & $; 1$ \\
\hline TA 10124 & Ae. tauschii & - & 1,2 & $1,1+$ & $1,2-, 3-$ & $; 1$ \\
\hline TA 10147 & Ae. tauschii & - & 4 & $;, 1 \mathrm{~N}$ & $;, 1 \mathrm{~N}$ & $1-, 2$ \\
\hline TA 10171 & Ae. tauschii & - & ; & ; & 0 ; & 0 \\
\hline TA 10206 & Ae. tauschii & - & $3+$ & $; 1$ & $1,3-\mathrm{Z}$ & $; 1$ \\
\hline CIae 15 & Ae. tauschii & - & $2+, 3$ & $1,2-, ;$ & 4 & $3+, 4$ \\
\hline
\end{tabular}

$\dagger \quad$ Infection types rated on a 0 (immune) to 4 (susceptible) scale where ';' denotes hypersensitive flecking, ' $\mathrm{N}$ ' denotes necrosis, ' $\mathrm{C}$ ' denotes chlorosis, and ' + ' and '-' signs indicate more or less sporulation, respectively compared to the standard infection type (Stakman et al., 1962). For accessions heterogeneous for infection types among plants, a '/' was used to separate different infection types corresponding to different plants. Symbol ',' was used to separate different infection types present on the same leaf. 
Table 5. Pair-wise comparisons of the association between reactions to the six races

\begin{tabular}{|c|c|c|c|c|}
\hline \multicolumn{2}{|c|}{ Association between } & \multirow[b]{2}{*}{$\chi^{2}$} & \multirow{2}{*}{$\begin{array}{l}\text { Association } \\
\text { Type } \dagger\end{array}$} & \multirow[b]{2}{*}{$P$ value: } \\
\hline Race & Race & & & \\
\hline TRTTF & TTKSK & 150.65 & + & $1.91 \mathrm{E}-32$ \\
\hline TRTTF & TTTTF & 59.68 & + & $6.87 \mathrm{E}-13$ \\
\hline TRTTF & QTHJC & 86.72 & + & $1.11 \mathrm{E}-18$ \\
\hline TRTTF & RKQQC & 37.024 & + & 4.55 E-08 \\
\hline TRTTF & TPMKC & 114.27 & + & 1.32 E-24 \\
\hline TTKSK & TTTTF & 36.77 & + & 5.16 E-08 \\
\hline TTKSK & QTHJC & 92.34 & + & 6.89 E-20 \\
\hline TTKSK & RKQQC & 63.65 & + & $9.76 \mathrm{E}-14$ \\
\hline TTKSK & TPMKC & 138.93 & + & $6.42 \mathrm{E}-30$ \\
\hline TTTTF & QTHJC & 129.32 & + & $7.59 \mathrm{E}-28$ \\
\hline TTTTF & RKQQC & 6.04 & ns & 0.11 \\
\hline TTTTF & TPMKC & 37.44 & + & $3.72 \mathrm{E}-08$ \\
\hline QTHJC & RKQQC & 51.98 & + & 3.02 E-11 \\
\hline QTHJC & TPMKC & 130.32 & + & $4.61 \mathrm{E}-28$ \\
\hline RKQQC & TPMKC & 101.31 & + & 8.13 E-22 \\
\hline
\end{tabular}

$\dagger \quad$ For significant associations, ' + ' indicates a positive association among resistant accessions and '-' indicates a negative association among resistant accessions. Symbol 'ns' indicates a non-significant association.

$\$ \quad$ Analyses performed with Microsoft Excel, critical $P$ value $=0.05$ 
Supplementary Table S1. Seedling infection types of Aegilops tauschii accessions to six races of Puccinia graminis f. sp. tritici 